\title{
Deformation and deceleration of coronal wave
}

\author{
Z. K. Xue ${ }^{1,3}$, Z. Q. Qu ${ }^{1}$, X. L. Yan ${ }^{1,2}$, L. Zhao ${ }^{4}$, and L. $\mathrm{Ma}^{1,3}$ \\ ${ }^{1}$ National Astronomical Observatories/Yunnan Astronomical Observatory, Chinese Academy of Sciences, Kunming, \\ 650011 Yunnan, PR China \\ e-mail: zkxue@ynao.ac.cn \\ 2 Key Laboratory of Solar Activity, National Astronomical Observatories, Chinese Academy of Sciences, 100012 Beijing, PR China \\ 3 University of Chinese Academy of Sciences, 100049 Beijing, PR China \\ 4 Yunnan Normal University, 650092 Kunming Yunnan, PR China
}

Received 14 November 2012 / Accepted 21 June 2013

\section{ABSTRACT}

\begin{abstract}
Aims. We studied the kinematics and morphology of two coronal waves to better understand the nature and origin of coronal waves. Methods. Using multi-wavelength observations of the Atmospheric Imaging Assembly (AIA) on board the Solar Dynamics Observatory (SDO) and the Extreme Ultraviolet Imager (EUVI) on board the twin spacecraft Solar-TErrestrial RElations Observatory (STEREO), we present morphological and dynamic characteristics of consecutive coronal waves on 2011 March 24. We also show the coronal magnetic field based on the potential field source surface model.

Results. This event contains several interesting aspects. The first coronal wave initially appeared after a surge-like eruption. Its front was changed and deformed significantly from a convex shape to a line-shaped appearance, and then to a concave configuration during its propagation to the northwest. The initial speeds ranged from $947 \mathrm{~km} \mathrm{~s}^{-1}$ to $560 \mathrm{~km} \mathrm{~s}^{-1}$. The first wave decelerated significantly after it passed through a filament channel. After the deceleration, the final propagation speeds of the wave were from $430 \mathrm{~km} \mathrm{~s}^{-1}$ to $312 \mathrm{~km} \mathrm{~s}^{-1}$. The second wave was found to appear after the first wave in the northwest side of the filament channel. Its wave front was more diffused and the speed was around $250 \mathrm{~km} \mathrm{~s}^{-1}$, much slower than that of the first wave.

Conclusions. The deformation of the first coronal wave was caused by the different speeds along different paths. The sudden deceleration implies that the refraction of the first wave took place at the boundary of the filament channel. The event provides evidence that the first coronal wave may be a coronal MHD shock wave, and the second wave may be the apparent propagation of the brightenings caused by successive stretching of the magnetic field lines.
\end{abstract}

Key words. Sun: activity - Sun: corona - Sun: magnetic topology - waves

\section{Introduction}

In the solar chromosphere, Moreton waves first observed in the early 1960s (Moreton 1960; Athay \& Moreton 1961) propagate with speeds ranging from $500 \mathrm{~km} \mathrm{~s}^{-1}$ to $2000 \mathrm{~km} \mathrm{~s}^{-1}$. They were interpreted as the chromospheric manifestations of magnetohydrodynamic (MHD) fast-mode shocks propagating in the corona (Uchida 1968). The wave-like propagations in the corona were first reported by Moses et al. (1997) and Thompson et al. (1998) with the observations of the Extreme-ultraviolet Imaging Telescope (EIT; Delaboudinière et al. 1995) on board the Solar and Heliospheric Observatory (SOHO). The coronal waves propagate away from the source across a significant area on the solar disk. They are followed by expanding dimming regions (Thompson et al. 1998, 1999). Since the discovery, a lot of coronal wave events have been studied in the past 15 years. On the other hand, it is widely accepted that the propagation speeds of coronal waves are lower than those of Moreton waves. Klassen et al. (2000) found using a statistical study based on EIT observations in 1997 that the speeds vary from $138 \mathrm{~km} \mathrm{~s}^{-1}$ to $465 \mathrm{~km} \mathrm{~s}^{-1}$ with an average of $271 \mathrm{~km} \mathrm{~s}^{-1}$. A large range of the coronal wave speeds, from 50 to $700 \mathrm{~km} \mathrm{~s}^{-1}$, was also reported by Thompson \& Myers (2009). It has been shown in many papers that coronal waves decelerate during their propagations (Warmuth et al. 2001; Veronig et al. 2011; Patsourakos \& Vourlidas 2009). Some authors have reported that the coronal waves stop at the boundary of coronal holes
(Thompson et al. 1999). When the coronal waves pass through the active regions, they can be reflected/refracted (Ofman \& Thompson 2002; Veronig et al. 2006). Biesecker et al. (2002) found that coronal waves are related to coronal mass ejections (CMEs), rather than flares by using statistical methods. With the observations of Geostationary Operational Environmental Satellite (GOES), EIT, and the Large Angle and Spectrometric Coronagraph (LASCO), Chen (2006) studied more than ten flares, some of which are accompanied by CMEs, to clarify the relationship between coronal waves and CMEs/flares. His result indicates that no matter how strong the associated flare is, a coronal wave can be observed only if a CME is present. Zheng et al. (2011) found that the speed of a coronal wave was higher than the spreading speed of a mini-CME, and thus distinguished the coronal wave from the mini-CME. Zheng et al. (2012a) observed that a blowout jet could trigger a coronal wave. Coronal waves can be observed at multiple channels using many instruments (Long et al. 2008; Shen \& Liu 2012a; Zheng et al. 2012b), such as EIT, the Extreme Ultraviolet Imager (EUVI; Howard et al. 2008) on board the twin spacecraft Solar-TErrestrial RElations Observatory (STEREO; Kaiser et al. 2008), and the Atmospheric Imaging Assembly (AIA; Lemen et al. 2012) on board the Solar Dynamics Observatory (SDO; Pesnell et al. 2012).

The nature of coronal waves has been debated for decades, and there has been no widely accepted interpretation. Based on MHD simulations, coronal waves are explained as fast-mode waves by Wang (2000) and Wu et al. (2001). They found that 
fast-mode waves are consistent with the observational characteristics of coronal waves. Chen et al. $(2002,2005)$ proposed a magnetic field-line stretching model to understand the nature of coronal waves. In this model, coronal waves are the apparent spread brightenings of plasma that is compressed by successive stretching of the magnetic field lines overlying the eruption flux rope, rather than real waves. Some other models, for example, the successive reconnection model (Attrill et al. 2007), the slow-mode wave model (Wills-Davey et al. 2007), and the current shell model (Delannée et al. 2008) have been proposed to interpret coronal waves. The combinations of both a non-wave and a wave component have also been proposed to describe coronal waves (Zhukov \& Auchère 2004; Cohen et al. 2009; Liu et al. 2010; Downs et al. 2011).

The SDO/AIA instrument provides multi-channel solar images with high spatial resolution and high temporal resolution. This helps us to study coronal waves in detail. We present an intriguing event that includes a first coronal wave and a following wave occurring on 2011 March 24 to identify the nature of the coronal waves. In Sect. 2, we analyze the data observed by SDO/AIA and STEREO/EUVI. Analysis is given in Sect. 3, while summary and discussion are presented in Sect. 4.

\section{Observational data}

Data used in this paper are mainly from SDO/AIA; AIA is an array of four telescopes that together provide full-disk images of the solar atmosphere with $1.5^{\prime \prime}$ spatial resolution (4096 × 4096-pixel images) at ten UV and EUV wavelengths every 12 s. The $193 \AA$ bandpass can obtain images of corona and hot flare plasma with characteristic temperatures of $1.5 \times 10^{6} \mathrm{~K}$. The $211 \AA$ channel $\left(T=2 \times 10^{6} \mathrm{~K}\right)$ is designed to observe active-region corona. The $171 \AA$ bandpass image primarily reflects the upper transition region/quiet corona material with an average formation temperature $T=6.5 \times 10^{5} \mathrm{~K}$. The images of the chromosphere and transition region are provided by the $304 \AA$ channel $\left(T=5 \times 10^{4} \mathrm{~K}\right)$. We also display other $304 \AA$ images observed by STEREO-B/EUVI to identify an eruptive surge.

All images are individually rotated to a reference time (12:00:00 UT) to correct for solar rotation. To show the coronal waves more clearly, we use running difference images to display the process of the coronal waves. They were produced by subtracting images $24 \mathrm{~s}$ earlier. The coronal waves can be observed at four AIA passbands (193, 211, 171, and $304 \AA$ ). However, they are most obvious in AIA $193 \AA$ images. Thus the AIA $193 \AA$ images were chosen as the first candidate to study the propagation process of the two coronal waves and their observational characteristics.

\section{Analysis}

\subsection{Dynamics of the surge-like eruption}

On 2011 March 24, a GOES M1.0 class flare occurred in National Oceanic and Atmospheric Administration (NOAA) active region 11176 located at the southeast of the solar disk. It began at 12:01 UT, peaked at 12:07 UT, and ended at 12:11 UT. A surge-like plasma motion started to erupt at about 12:04 UT in the same active region. It seems that the flare triggered the surge eruption. Figure 1 shows the shape of the surge-like plasma motion during the eruption with two different angles of view. The top panels are observed at the $304 \AA$ channel of SDO/AIA
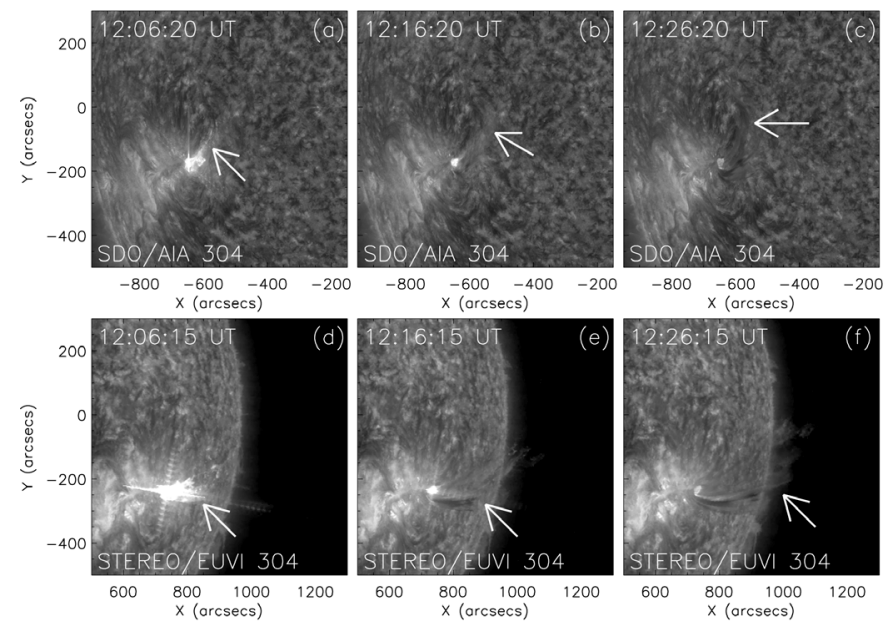

Fig. 1. Surge-like eruption observed by SDO/AIA (upper panels) and STEREO-B/EUVI (lower panels). The white arrows point to the surge.

and the bottom panels at $304 \AA$ of STEREO-B/EUVI. From panels a-c, the eruptive surge cannot be clearly observed; it appeared as a slightly curved dark feature that erupted to the northwest. Instead in panels $\mathrm{d}-\mathrm{f}$, marked by white arrows, one can see that the surge erupted intensively. The eruptive surge showed a triangular shape slightly curved to the north. It is the reason why we need to observe the Sun from various viewing angles, as STEREO-A and STEREO-B do. The surge decayed around 12:47 UT. According to the SOHO LASCO observation, an associated CME was first seen in the C2 coronagraph at 12:48 UT with a speed of $540 \mathrm{~km} \mathrm{~s}^{-1}$ and appeared in the $\mathrm{C} 3$ coronagraph at 13:42 UT.

\subsection{First coronal wave and second coronal wave}

The morphological evolution of the first coronal wave in the AIA $193 \AA$ running difference images is illustrated in Fig. 2. The wave front can be identified clearly in these images and positioned by the white arrows. The flare position is marked by the black arrow in Fig. 2a. The wave first appeared at about 12:05 UT. In general, coronal/Moreton waves are triggered from one source point (also called the radiant point) and propagate in all directions around the radiant point (Veronig et al. 2008; Balasubramaniam et al. 2010). When first observed this wave did not appear to generate from an original/radiant point, but with a convex bright front as shown in Fig. 2b. Then the coronal wave propagated from its original position to the northwest of the solar disk along a very narrow path rather than in all directions (as reported by Thompson et al. 1998; Long et al. 2008; Patsourakos \& Vourlidas 2009). At the same time, the eruptive surge expanded slowly. The spatial separation between the first coronal wave and the eruptive surge indicates that the surge-like eruption is associated with the wave's generation.

The shape of the first wave front changed significantly during its propagation. The curvature of the wave front became gradually smaller, and around 12:15 UT, the bright front took on a straight-line structure (Fig. 2d). And as the wave went forward, its shape then changed to a concave structure (Fig. 2e). The intensity of the wave front dissipated and disappeared at about 12:30 UT. The visible propagation lasted for $25 \mathrm{~min}$.

A filament channel was in the path of the spreading wave (marked by the arrow in Fig. 3), and it was almost perpendicular to the wave propagation path. It took an appearance of a 

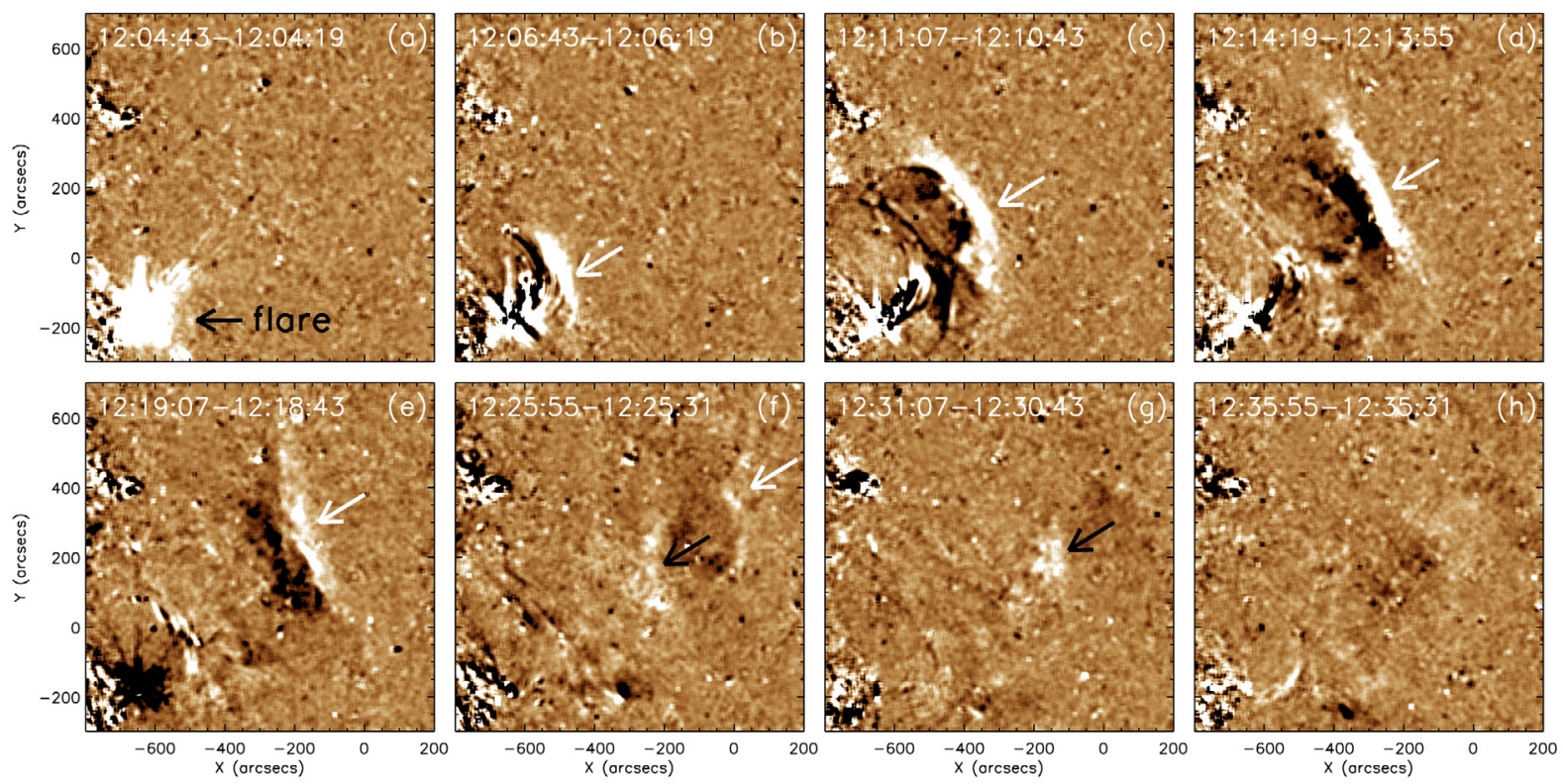

Fig. 2. Evolution of two coronal waves in the AIA $193 \AA$ running difference images. The position of the flare is marked by the black arrow in panel a). The first and second waves are indicated by the white arrows in panels b) $-\mathbf{f}$ ) and black arrows in panels f)-g) respectively.

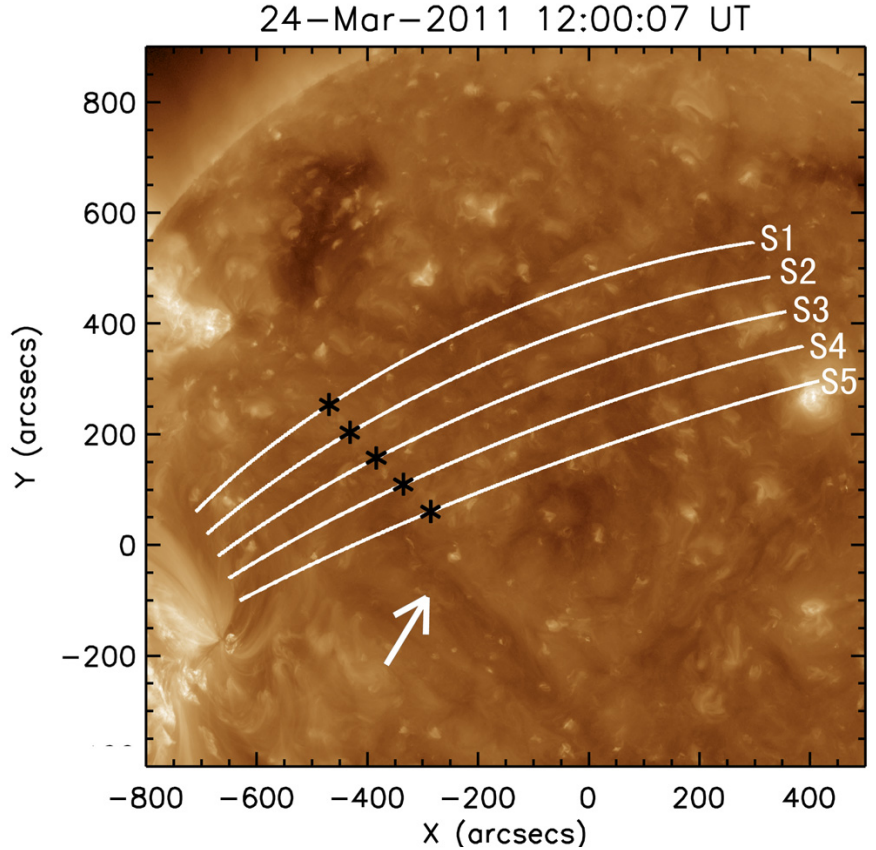

Fig. 3. Five slices in the AIA $193 \AA$ image. The filament channel is indicated by the white arrow. The positions where the deceleration of first wave happened are marked with black symbols asterisks.

linear dark region observed in the AIA $193 \AA$ images. A small filament was found in this filament channel observed in the AIA $304 \AA ̊$ Amage. At 12:19 UT, a second coronal wave appeared at the northwest edge of the filament channel and propagated to the northwest of the solar disk following the first wave. Its wave front was more diffused, indicated by the black arrows in Figs. 2f and $\mathrm{g}$. The second wave propagated within a narrower path over a shorter distance than the first wave. At 12:35 UT, the second wave disappeared from the $193 \AA$ images.
The evolutions of the first and second waves in AIA $211 \AA$, $171 \AA$, and $304 \AA$ running difference images are displayed in Fig. 4. The first row is arranged to show the running difference images of $211 \AA$ (Figs. 4a1-4a4), the second row corresponds to $171 \AA$ (Figs. $4 \mathrm{~b} 1-4 \mathrm{~b} 4$ ), and the third row to $304 \AA$ (Figs. 4c1-4c4). As displayed in Fig. 4, the first coronal wave could be also clearly seen in the AIA $211 \AA$ images, and it had similar characteristics to those observed in the $193 \AA$ images, such as the propagation direction and changing shape of the coronal wave front. In the $171 \AA$ A running difference images, the first wave front was bright at its first appearance, but then became dark (Wills-Davey \& Thompson 1999; Liu et al. 2010; Schrijver et al. 2011). In the $304 \AA$ images, the first wave was not clearly seen. It did not propagate far and disappeared quickly from the $304 \AA$ images. For the second coronal wave which is marked by the black arrows (Figs. $4 \mathrm{a} 4$ and $4 \mathrm{~b} 4$ ), it can be seen in the AIA $211 \AA$ and $171 \AA$ images and is not visible in the $304 \AA$ Amages. Comparisons between these channels signify that the waves propagated dominantly in the corona and left a weak trace in the chromosphere according to the formation depths of these bandpasses.

To study the kinematics of the coronal waves, we chose five slices along the wave propagation path to show the time-distance diagrams (Fig. 5). In Fig. 3, these slices are indicated by white solid lines and labeled by S1-S5 from the upper left to the lower right. The slices are extracted from the active region. Since the first coronal wave appeared with an arch-shape rather than from a central point, they are selected along the wave propagation path and perpendicular to the wave front. They are along the solar surface with a width of $435 \mathrm{~km}$ and a length of $8.7 \times 10^{5} \mathrm{Km}$ and the distances determined in 2D images are corrected to the distances in 3D images. We fit the time-distance diagrams with linear functions and thus obtain the speeds of the coronal waves. The fitting results are shown in Fig. 5 and are indicated by different color lines. Figures $5 \mathrm{a}-5 \mathrm{e}$ is obtained from the $193 \AA$ running difference images, corresponding to the five slices S1-S5, respectively. As shown in Fig. 5a, the first wave propagated with 

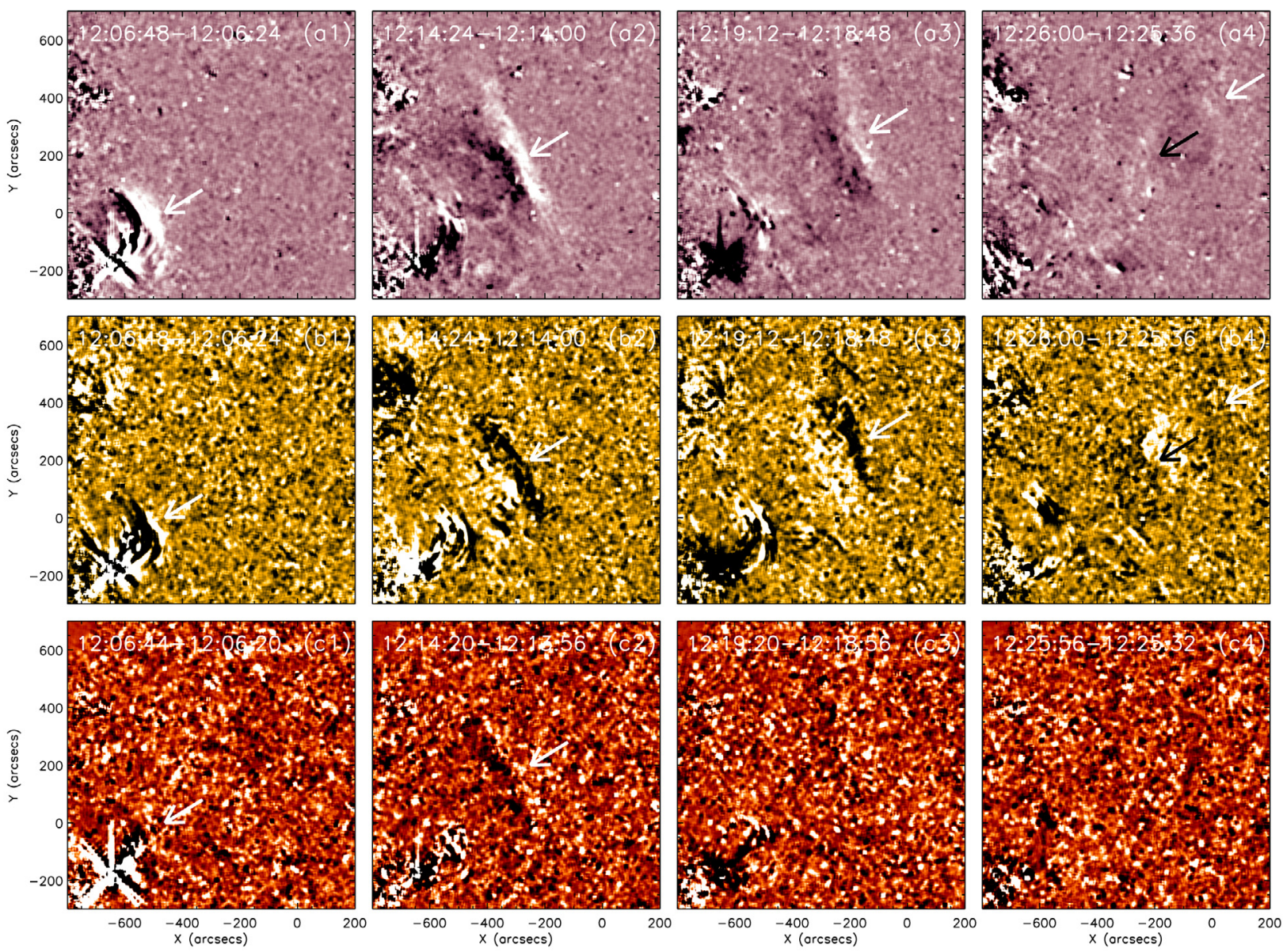

Fig. 4. Evolution of two coronal waves in the AIA $211 \AA$, $171 \AA$, and $304 \AA$ running difference images. The first and second waves are indicated by the white arrows and black arrows, respectively.
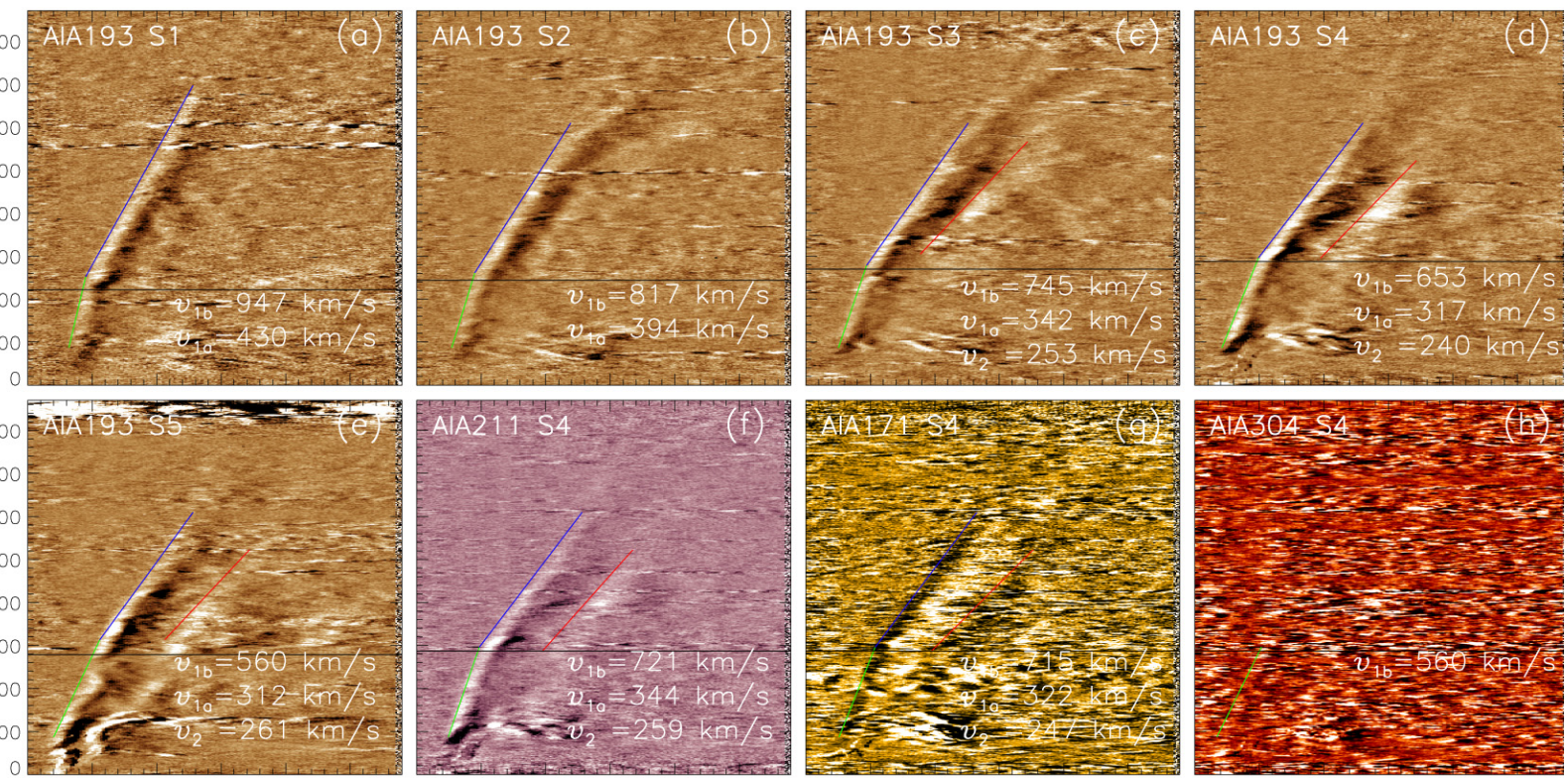

Fig. 5. Time-distance diagrams of two coronal waves. Panels a)-e) are obtained from the $193 \AA$ running difference images along slices S1-S5, respectively. Panels f)-h) are obtained from the $211 \AA$, $171 \AA$, and $304 \AA$ running difference images, respectively, along slice S4. They are fitted with linear functions, and the fitting results are indicated by different color lines. The speeds of the first wave before and after the filament channel are shown in panels with $v_{1 \mathrm{~b}}$ (the slopes of the green lines) and $v_{1 \mathrm{a}}$ (the slopes of the blue lines). The speeds of the second wave are shown with $v_{2}$ (the slopes of the red lines). The northwest edge of the filament channel is marked by black lines. 
an initial speed of $947 \mathrm{~km} \mathrm{~s}^{-1}$ (denoted with $v_{1 \mathrm{~b}}$ ) along $\mathrm{S} 1$. At about 12:10 UT, when it passed through the filament channel (indicated by the black lines), its propagation speed quickly decreased to $430 \mathrm{~km} \mathrm{~s}^{-1}$ (denoted with $v_{1 \mathrm{a}}$ ) and this speed remained until it was no longer observed. The same behavior is observed along S2-S5 when the wave passed through the filament channel. The initial speeds of the first wave along the S2-S5 were $817,745,653$, and $560 \mathrm{~km} \mathrm{~s}^{-1}$, respectively, and the final speeds dropped to $394,342,317$, and $312 \mathrm{~km} \mathrm{~s}^{-1}$, respectively. The initial speeds were almost twice the final speeds. The speeds decreased from S1 to S5 at the same time.

To compare with the result calculated from the $193 \AA$ images, we obtained the time-distance diagrams along slice S4 from the 211, 171, and $304 \AA$ running difference images and show them in Figs. $5 f-5 h$, respectively. The first coronal wave also showed a quick deceleration when it passed through the filament channel observed at the 211 and $171 \AA$ A channel. The initial speeds were 721 and $715 \mathrm{~km} \mathrm{~s}^{-1}$, respectively, and the final speeds were 344 and $322 \mathrm{~km} \mathrm{~s}^{-1}$, respectively obtained from the $211 \AA$ and $171 \AA$ running difference images. In the $304 \AA$ images, the intensity of the first wave was so weak that we can only see the wave before it passed through the filament channel. The initial speed of the first wave along S4 was found to be $560 \mathrm{~km} \mathrm{~s}^{-1}$ at the $304 \AA$ channel, smaller than those observed in the $193 \AA, 211 \AA$, and $171 \AA$ images.

The kinematics of the second coronal wave was also studied with the time-slice images. We do not find any trace of the second wave following the first wave along slices S1 and S2. The second wave is clearly visible in Figs. $5 c-5$ e. The speeds of this wave (denoted with $v_{2}$ ) along slices S3-S5 are 253, 240, and $261 \mathrm{~km} \mathrm{~s}^{-1}$, respectively, in the $193 \AA$ images. The second wave can be also seen in the $211 \AA$ and $171 \AA$ images (Figs. 5f and $5 \mathrm{~g}$ ), but it is not visible in the $304 \AA$ images. From the $211 \AA$ and $171 \AA$ images, we obtained the speeds of the second wave, 259 and $247 \mathrm{~km} \mathrm{~s}^{-1}$, respectively.

\subsection{Formation of coronal waves}

The nature of coronal waves is not yet completely understood. Chen et al. $(2002,2005)$ proposed a field-line stretching model to explain the physical nature of coronal waves. In their model, a fast-mode shock wave (also known as a coronal Moreton wave) is first piston-driven by an erupting flux rope, and a so-called EIT wave appears to move the density enhancement that is compressed by the successive stretching magnetic field lines, but it is not a real wave. They assumed simply that if all field lines are concentric semicircles, the fast-mode wave should be about three times the EIT wave.

To investigate the coronal magnetic topology where the waves propagated, we extrapolated the coronal magnetic field by using the potential field source surface (PFSS; Schrijver \& Rosa 2003) model. The coronal magnetic topology is shown in Fig. 6, overlapped on the AIA $193 \AA$ image. Some of the magnetic loops lay on the filament channel. Based on the field-line stretching model (Chen et al. 2002, 2005) and the coronal magnetic topology (Fig. 6), we show a series of drawings to explain our observational results (Fig. 7). The surge erupted (indicated by red circle) at time $t_{1}$, and the innermost magnetic field line was pushed and stretched at the top. A perturbation appeared with a form of a piston-driven shock and propagated freely in the corona (indicated by pink lines), corresponding to the first coronal wave in this paper.

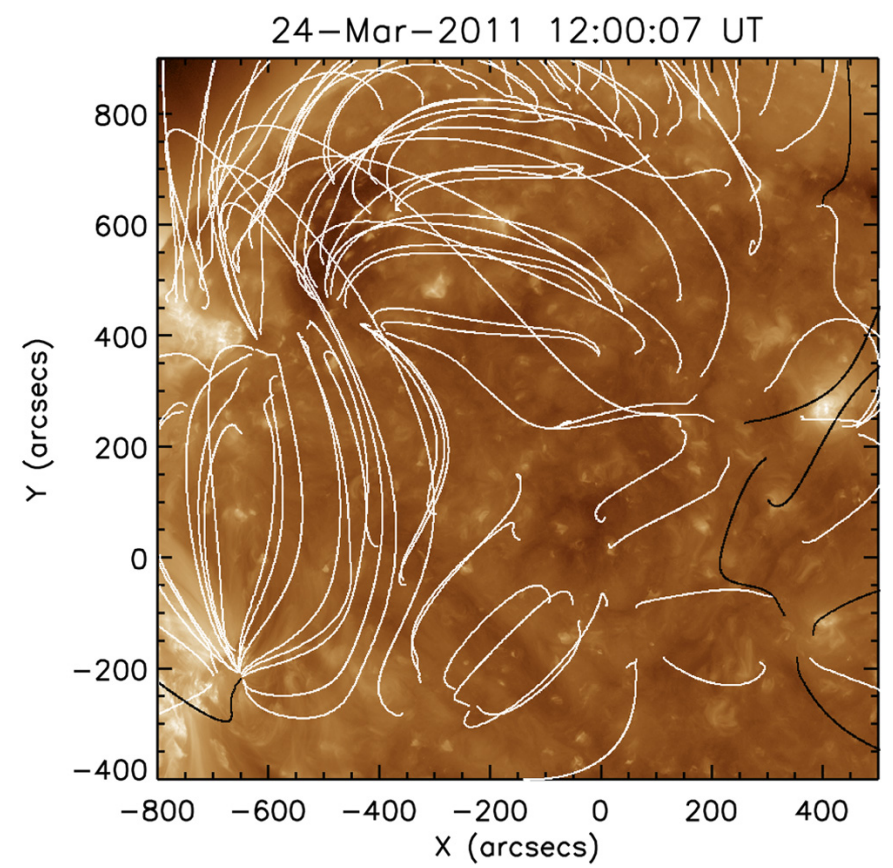

Fig. 6. PFSS extrapolation. It is overlapped on the AIA $193 \AA$ image before the waves. The white lines represent closed magnetic field lines, and the black lines represent open magnetic field lines.

Then the perturbation of the field line was transferred to the footpoint. Meanwhile, the top of the upper field line was pushed and stretched $\left(t=t_{2}\right)$. An EIT wave appeared at the footpoint (marked by the green lines). The magnetic field lines ascended one by one $\left(t=t_{3}\right)$. At time $t=t_{4}$, the larger magnetic field lines crossed the filament channel, but the EIT wave did not pass through the filament channel, neither did it appear in the filament channel. The result was that the EIT wave stopped at the magnetic separatrix $\left(t=t_{4}\right)$. At $t=t_{5}$, the larger loops over the active region and the filament channel were pushed and stretched, while the EIT wave appeared again at their footpoints. And then the EIT wave spread in the corona $\left(t=t_{6}\right)$.

\section{Summary and discussion}

Based on observations from SDO/AIA and STEREO-B/EUVI on 2011 March 24, we present two consecutive coronal waves along similar paths after an M1.0 flare and a surge-like eruption. Their different morphologies and kinematics are studied. We summarize the observational results as follows:

1. The front of the first coronal wave changed from a convex shape to a line-shaped appearance as it propagated, and finally to a concave shape;

2. The speeds of the first wave along different propagation paths were different. The speeds along five selected slices were $947,817,745,653$, and $560 \mathrm{~km} \mathrm{~s}^{-1}$, respectively, at the start time, obtained from the $193 \AA$ images;

3. After the first coronal wave passed through the filament channel, it decelerated suddenly. The final speeds of the first wave ranged from $430 \mathrm{~km} \mathrm{~s}^{-1}$ to $312 \mathrm{~km} \mathrm{~s}^{-1}$ along the five slices. The mean ratio of the initial speeds $v_{1 \mathrm{~b}}$ to the final speeds $v_{1 \mathrm{a}}$ was about 2 ;

4. The second coronal wave was observed to propagate with an average speed of $250 \mathrm{~km} \mathrm{~s}^{-1}$ after the first wave passed through the filament channel. 

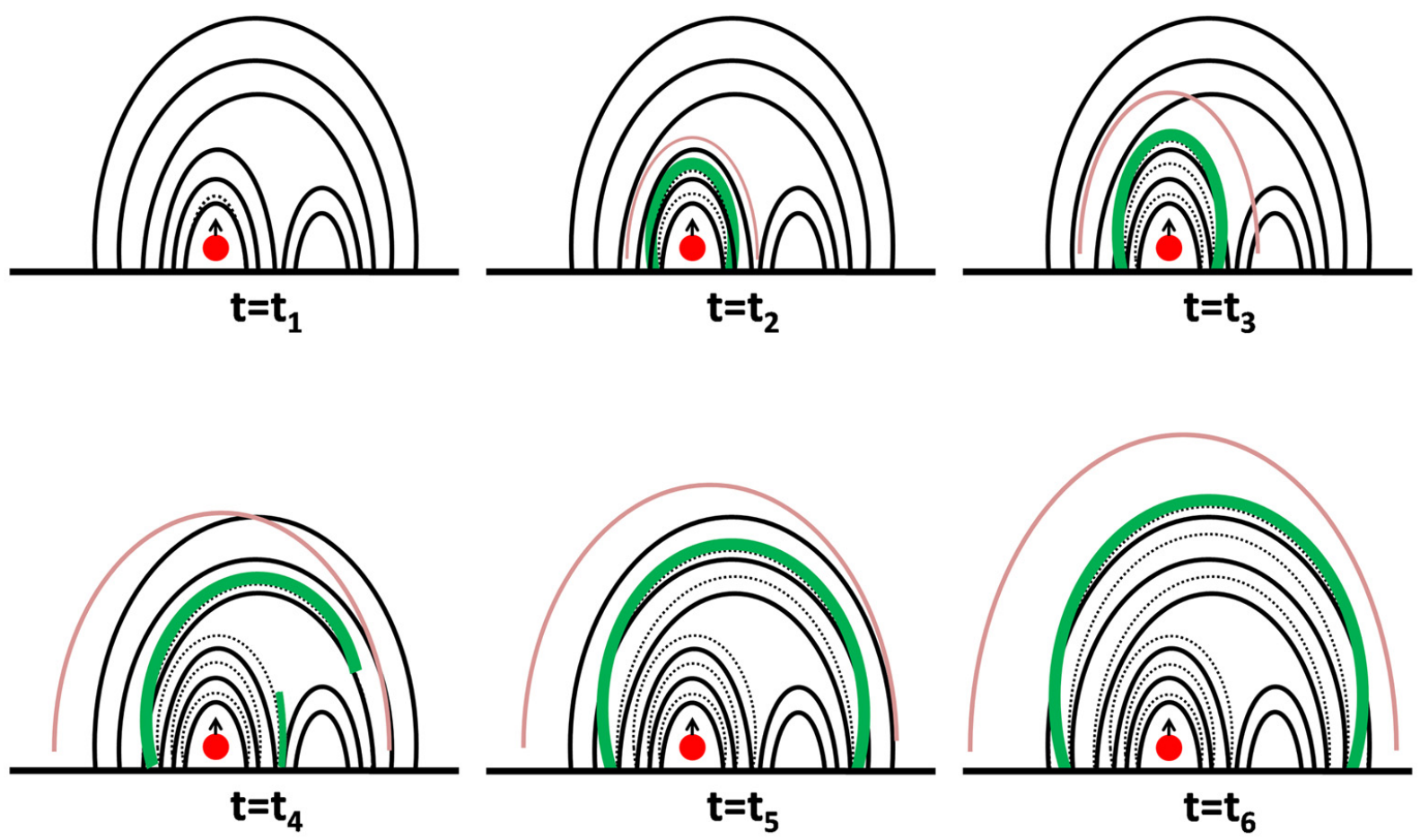

Fig. 7. Drawings based on the field-line stretching model and the coronal magnetic topology. The eruptive surge is marked by the red circle. The magnetic field lines before perturbation are indicated by the black solid lines and those after perturbation are indicated by the black dotted lines. The pink lines represent the piston-driven shock. The typical EIT waves are shown as green lines.

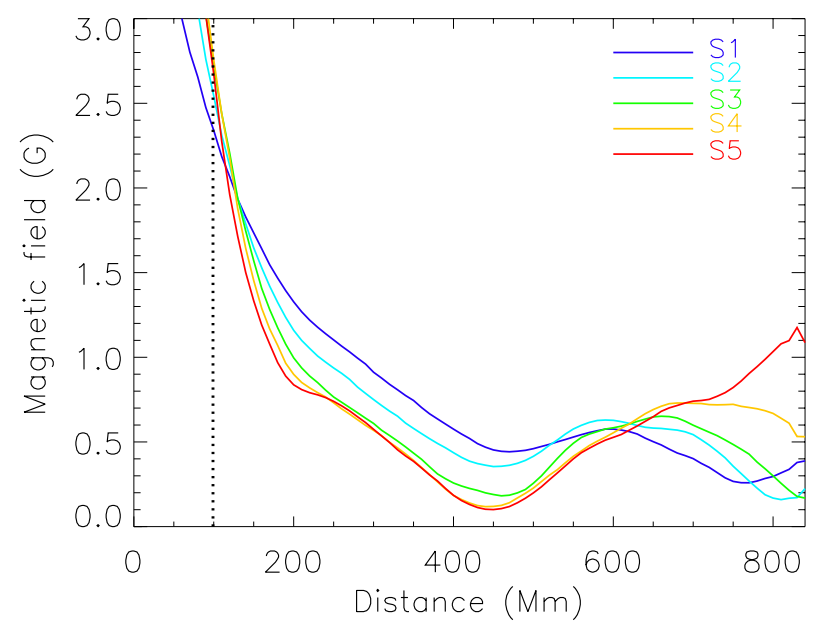

Fig. 8. Coronal magnetic field strengths along the five slices (S1-S5) using the PFSS model at a height of $0.1 R_{\text {Sun }}$ above the solar surface and indicated by different color lines. The start time of the first wave is marked by the black dotted line.

The northeast part of the first coronal wave front propagated faster than the southwest part. If we select the southwest part of the wave as a reference, it is easily found that the wave front rotated clockwise. The coronal magnetic field strengths along the five slices (S1-S5) are shown in Fig. 8 with different color lines. They are obtained from the PFSS model at a height of $0.1 R_{\text {Sun }}$ above the solar surface (Patsourakos et al. 2009; Kienreich et al. 2009). It is likely that the magnetic field strengths along the northeast path were stronger than those along the southwest path. This may be the cause of the different initial speeds of different parts of the first wave. The shape change of the first wave front may be caused by the different speeds along these paths. When the northeast part of the wave went behind the southwest part, the wave front showed a convex structure. When the northeast part of the wave caught up the southwest part, it appeared as a line-shaped structure. Finally, when the northeast part of the wave exceeded the southwest part, the wave front became a concave structure.

Decelerations of coronal waves have been found by many researchers. Warmuth et al. (2001) reported a non-linear speed profile of a coronal wave, and there was an acceleration at least during the first $2000 \mathrm{~s}$ of the observations. Patsourakos \& Vourlidas (2009) found that the wave had a non-zero deceleration of $25 \mathrm{~m} \mathrm{~s}^{-2}$ with a quadratic fit. The result obtained by Long et al. (2008) revealed that the wave front was accelerated away from the flare site with maximal acceleration of $816 \mathrm{~m} \mathrm{~s}^{-2}$ before it decelerated by $413 \mathrm{~m} \mathrm{~s}^{-2}$; these velocities were calculated from the $171 \AA$ images. Using the observation of Hinode/EIS, a strong deceleration of $540 \mathrm{~m} \mathrm{~s}^{-2}$ of a coronal wave front was calculated by Veronig et al. (2011). Warmuth \& Mann (2011) analyzed the relationship between the initial speeds and the accelerations by a statistical method. Their result revealed that the larger the initial speeds were, the more significant the accelerations were. However, in our case, the deceleration of the first wave was different. The first wave decelerated suddenly, rather than gradually. Before and after the deceleration, the first coronal wave propagated with constant speeds.

The positions where the deceleration of the first coronal wave occurred are marked with the black asterisks in Fig. 3 along the five slices. They are located on the northwest side of the filament channel. Wang (2000) and Ofman \& Thompson (2002) used MHD simulations and proposed that coronal waves could be refracted by active regions. Shen $\&$ Liu (2012b) used observational data from SDO and found that coronal waves might be refracted by bright points. The refraction of coronal waves at bright coronal structures (small-scale, local loops) was reported 
by Li et al. (2012). For our observational results, we propose that the first wave was refracted at the boundary of the filament channel, thus the speed of the first wave changed suddenly to lower one.

It is also found that the second coronal wave front was more diffused and propagated more slowly with a speed of about $250 \mathrm{~km} \mathrm{~s}^{-1}$. The mean ratio of $v_{1 \mathrm{~b}}$ to $v_{2}$ is 2.7 . Many authors observed wave pairs at the same time. Chen and Wu (2011) observed a pair of waves during a wave event and they explained that the first wave is a coronal Moreton wave and the second slow wave is an EIT wave rather than a real wave. Shen \& Liu (2012a) reported another pair. They proposed that the first wave is a fast magneto-sonic shock wave and the slower pseudowave is caused by the reconfiguration of the low coronal magnetic fields. The observations of the co-existence of wave pairs were analyzed by Kumar et al. (2013). They concluded that the faster wave is a coronal Moreton wave and the second wave is classical EIT wave. Liu et al. (2011) reported quasi-periodic fast-propagating magnetosonic waves. They found that multiple arc-shaped wave trains occurred consecutively and that the wave trains had similar amplitudes, wavelengths, and propagation speeds. Ofman et al. (2011) successfully simulated the multiple arc-shaped wave trains reported by Liu et al. (2011). Liu et al. (2012) observed that quasi-periodic wave trains within a broad global coronal wave propagated coherently. These wave trains were repeatedly launched ahead of the lateral CME front with a 2 min period. They appeared as successive arc-shaped pulses and propagated with a speed up to $1400 \mathrm{~km} \mathrm{~s}^{-1}$.

According to the scenario shown in Sect. 3.3, a pair of coronal waves can be observed in one wave event. We suggest that the first coronal wave in our event may be a coronal MHD shock wave triggered by the surge-like eruption. The second coronal wave may be a typical EIT wave as Chen et al. (2002, 2005) concluded. The speed of the second coronal wave was nearly a third of the initial speed of the first wave. This was consistent with the conclusions of Chen et al. $(2002,2005)$ that the speed of the shock should be about three times that of the EIT wave if the coronal magnetic field lines are semicircles.

Acknowledgements. We would like to express our gratitude to the referee for constructive comments and thank the SDO/AIA and STEREO/EUVI teams for data support. This work is sponsored by the National Science Foundation of China (NSFC) under grant numbers 10903027, 11078005, 10943002, 11103075, Key Laboratory of Solar Activity of CAS under number KLSA201303, China's 973 project under grant number G2011CB811400.

\section{References}

Athay, R. G., \& Moreton, G. E. 1961, ApJ, 133, 935

Attrill, G. D. R., Harra, L. K., van Driel-Gesztelyi, L., et al. 2007, ApJ, 656, L101

Balasubramaniam, K. S., Cliver, E. W., Pevtsov, A., et al. 2010, ApJ, 723, 587

Biesecker, D. A., Myers, D. C., Thompson, B. J., et al. 2002, ApJ, 569, 1009

Chen, P. F. 2006, ApJ, 641, L153

Chen, P. F., \& Wu, Y. 2011, ApJ, 732, L20

Chen, P. F., Wu, S. T., Shibata, K., \& Fang, C. 2002, ApJ, 572, L99

Chen, P. F., Fang, C., \& Shibata, K. 2005, ApJ, 622, 1202

Cohen, O., Attrill, G. D. R., Manchester, W. B., et al. 2009, ApJ, 705, 587

Delaboudinière, J. P., Artzner, G. E., Brunaud, J., et al. 1995, Sol. Phys., 162, 291

Delannée, C, Török, T., Aulanier, G., et al. 2008, Sol. Phys., 247, 123

Downs, C., Roussev, I. I., van der Holst, B., et al. 2011, ApJ, 728, 2

Howard, R. A., Moses, J. D., Vourlidas, A., et al. 2008, Space Sci. Rev., 136, 67

Kaiser, M. L., Kucera, T. A., Davila, J. M., et al. 2008, Space Sci. Rev., 136. 5

Kienreich, I. W., Temmer, M., \& Veronig, A. M. 2009, ApJ, 703, L118

Klassen, A., Aurass, H., Mann, G., et al. 2000, A\&AS, 141, 357

Kumar, P., Cho, K. S., Chen, P. F., et al. 2013, Sol. Phys, 282, 523

Lemen, J. R., Title, A. M., Akin, D. J., et al. 2012, Sol. Phys., 275, 17

Li, T., Zhang, J., Yang, S., et al. 2012, ApJ, 746, 13

Liu, W., Nitta, N. V., Schrijiver, C. J., et al. 2010, ApJ, 723, L53

Liu, W., Title, A. M., Zhao, J., et al. 2011, ApJ, 736, L13

Liu, W., Ofman, L., Nitta, N. V., et al. 2012, ApJ, 753, 52

Long, D. M., Gallagher, P. T., McAteer, B. T., et al. 2008, ApJ, 680, L81

Moreton, G. E. 1960, AJ, 65, 494

Moses, D., Clette, F., Delaboudinière, J.-P., et al. 1997, Sol. Phys., 175, 571

Ofman, L., \& Thompson, B. J. 2002, ApJ, 574, 440

Ofman, L., Liu, W., Title, A., \& Aschwanden, M. 2011, ApJ, 740, L33

Patsourakos, S., \& Vourlidas, A. 2009, ApJ, 700, L182

Patsourakos, S., Vourlidas, A., Wang, Y. M., et al. 2009, Sol. Phys., 259, 49

Pesnell, W. D., Thompson, B. J., \& Chamberlin, P. C. 2012, Sol. Phys., 275, 3

Schrijver, C. J., \& De Rose, M. L. 2003, Sol. Phys., 212, 165

Schrijver, C. J., Aulanier, G., Title, A. M., et al. 2011, ApJ, 738, 167

Shen, Y. D., \& Liu, Y. 2012a, ApJ, 752, L23

Shen, Y. D., \& Liu, Y. 2012b, ApJ, 754, 7

Thompson, B. J., \& Myers, D. C. 2009, ApJS, 183, 225

Thompson, B. J., Plunkett, S. P., Gurman, J. B., et al. 1998, Geophys. Res. Lett., 25,2465

Thompson, B. J., Gurman, J. B., Neupert, W. M., et al. 1999, ApJ, 517, L151

Uchida, Y. 1968, Sol. Phys., 4, 30

Veronig, A. M., Temmer, M., Vršnak, B., et al. 2006, ApJ, 647, 1466

Veronig, A. M., Temmer, M., \& Vršnak, B. 2008, ApJ, 681, L113

Veronig, A. M., Gömöry, P., Kienreich, I. W., et al. 2011, ApJ, 743, L10

Wang, Y. M. 2000, ApJ, 543, L89

Warmuth, A., \& Mann, G. 2011, A\&A, 532, A151

Warmuth, A., Vršnak, B., Aurass, H., \& Hanslmeier, A. 2001, ApJ, 560, L105

Wills-Davey, M. J., \& Thompson, B. J. 1999, Sol. Phys., 190, 467

Wills-Davey, M. J., DeForest, C. E., \& Stenflo, J. O. 2007, ApJ, 664, 556

Wu, S. T., Zheng, H., Wang, S., et al. 2001, J. Geophys. Rev., 106, 25089

Zheng, R. S., Jiang, Y. C., Hong, J. C., et al. 2011, ApJ, 739, L39

Zheng, R. S., Jiang, Y. C., Yang, J. Y., et al. 2012a, ApJ, 753, 112

Zheng, R. S., Jiang, Y. C., Yang, J. Y., et al. 2012b, A\&A, 541, A49

Zhukov, A. N., \& Auchère, F. 2004, A\&A, 427, 705 